\title{
RENDER SETTINGS IMPACT ANALYSES ON QUALITY OF COMPLEX 3D GRAPHIC STRUCTURE
}

\author{
SKALA, T.; MuZA, R. \& MrVaC, N.
}

Abstract: Computer graphic is a quite new area in graphic technology. Huge computer performances are used for rendering complex graphic content. $3 D$ is also relatively new and complex part of design in which we use a lot of information, both visual and multimedial. Through this work rendering procedure was analyzed. Time consuming for rendering (which depends on render settings) is one of the biggest problems. We had studied and measured duration of rendering a complex graphic scene, so we could find which settings to use for different purpose. Manipulating certain settings in the program would get us to different, more favorable time but also we changed the appearance of the scene. We have also found and displayed the optimum time to produce quality product. Today quality is very important in making graphic products, but there are situations where a greater importance is given to the speed of its development. Changing the settings is possible up to certain limits. After that limit quality of products decreases.

Key words: 3D modeling, image rendering, graphic structure, scene complexity
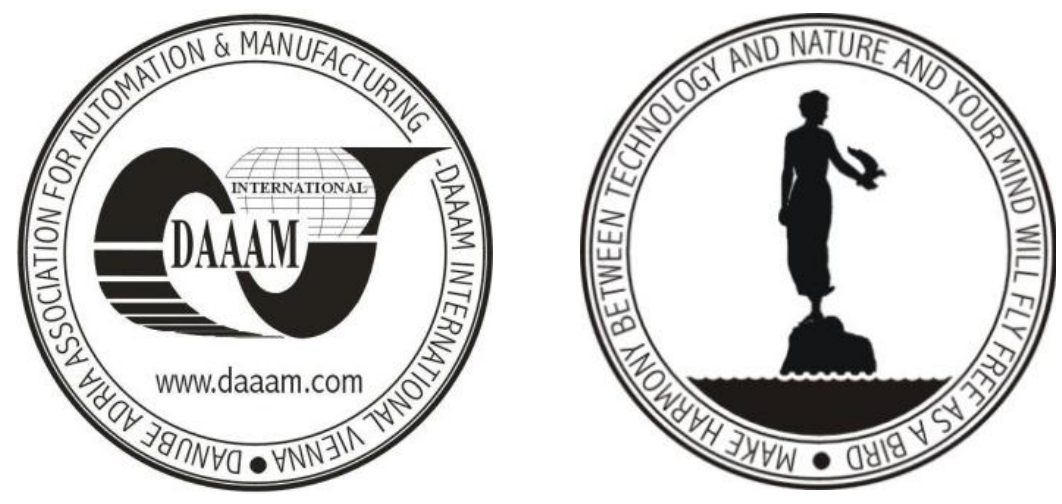

Authors' data: Dr. Sc. Skala, T[ibor]; Muza, R[obert]; Univ.Prof. Dr. Sc. Mrvac, N[ikola], University of Zagreb, Faculty of Graphic Arts, Getaldiceva 2, 10000 Zagreb, Croatia, tibor.skala@grf.hr, robert.muza@gmail.com, nikola.mrvac@grf.hr

This Publication has to be referred as: Skala, T[ibor]; Muza, R[obert] \& Mrvac, N[ikola] (2010). Render Settings Impact Analyses on Quality of Complex 3D Graphic Structure, Chapter 15 in DAAAM International Scientific Book 2010, pp. 141-150, B. Katalinic (Ed.), Published by DAAAM International, ISBN 978-3901509-74-2, ISSN 1726-9687, Vienna, Austria

DOI: $10.2507 /$ daaam.scibook.2010.15 


\section{Introduction}

Over the last few years we have seen widespread deployment of computer graphics in the many scientific fields such as architecture, film industry, medicine, design, marketing, etc. Some of these computer graphics are made for the purpose of better and easier understanding of the subject. In that case 3rd dimension is introduced. There is existence of very sophisticated computer programs made for 3D visualization and modeling, such as: 3D Studio Max, Maya, POV-Ray, Cinema 4D, Blender, Lightwave and others. While rendering graphic scene the biggest problem we encounter with was time consuming that depends on scene complexity. Often the complexity of the scene is defined as a number of polygons that made the scene. In this paper we will show how necessary it is to set parameters for obtaining the ideal and desired scene in the shortest period of time with the acceptable quality. Such studies improve and advance knowledge about the behavior of light within the scene, the optimal use of modern computer methods and contribute to accelerating the performance of the final product and reduce computing costs.

\section{Theoretic overview}

Modeling and visualization of 3D graphic structure is great achievement in computer technology and allows you to display three-dimensional objects and entire scenes. To show the 3D scene as an image or animation, it is necessary to render the scene. Rendering is the process of converting one model or the scene in a $2 \mathrm{D}$ image (video) using the particular program. In the graphic field it is the last step in which the final appearance of the model or animation occurs. Rendering would not be much needed when, but due to very large complex scenes, the graphics card that is the computer hardware is not able to calculate it (Skala, 2010).

\subsection{Methods of image creating and rendering}

The image is created using the visible part of the electromagnetic waves - light. In order to formally describe the propagation of light through space, and to define the theoretical light flow suitable for computer simulation, it is necessary to analyze and define the notion of light, a way of spreading light, and all the phenomena and effects that can occur (Arvo, 1995).

There are a number of different interpretations of light that have emerged over the years. The simplest understanding of light within the geometric optics, which states that light, is a set of light rays - the discrete, abstract structures without the weight and dimensions. On the other hand, in the framework of physical optics, light is understood as electromagnetic radiation and accordingly, the spread of light seen as expansion waves. Light "rays" are then nothing but an approximation of wave fronts of light. The third notion of light coming from quantum physics, where light is a set of elementary particles called photons. Currently the most complete model of perception of light is a combination of wave and particle interpretations of the nature of light. Regardless of the interpretation of the model, light shows certain properties and effects that occur when the spread of electromagnetic waves in the visible 
wavelength region. The image is created based on the basic properties of light, namely the reflection and refraction (Rigau et al., 2001).

The biggest benefit of rendering graphic structure is getting photorealistic images. It is done in a way to follow every ray of light in a scene which is very impractical, complex and requires a lot of time. However, there are four different mathematical algorithms that enable more efficient and easier to simulate realistic light. The first is the "rasterization", which includes "scanline rendering (scan row by row) of the images and its conversion from geometry into points or pixels. The second one is the "ray casting" - the beam of light are sent only for the visible part of the scene (from the perspective of the camera) and recorded only the scene geometry and basic optical laws such as the intensity of reflection (Gortler et al. 1993). The third is the "ray tracing" (monitoring beam of light) that is very similar to the "ray casting", but uses many advanced optical simulations. The fourth and last algorithm is "radiosity" which calculates the passage of light to the surface and its rejection, for the several times (depending on preference), while the beam of light reaches the camera.

\subsection{Photorealistic rendering}

Creating photorealistic images or the rendering process is a method of creating an image from a model using a special computer program (Wald \& Slusallek, 2001). The program creates a model that represents a description of three dimensional objects in the defined language or data structure. The model contains information on the geometry, the point of view, texture and lighting information. Rendering is also used in describing the process of calculating the special effects in video processing. Primarily used in computing, in simulators, movies and special effects, then design

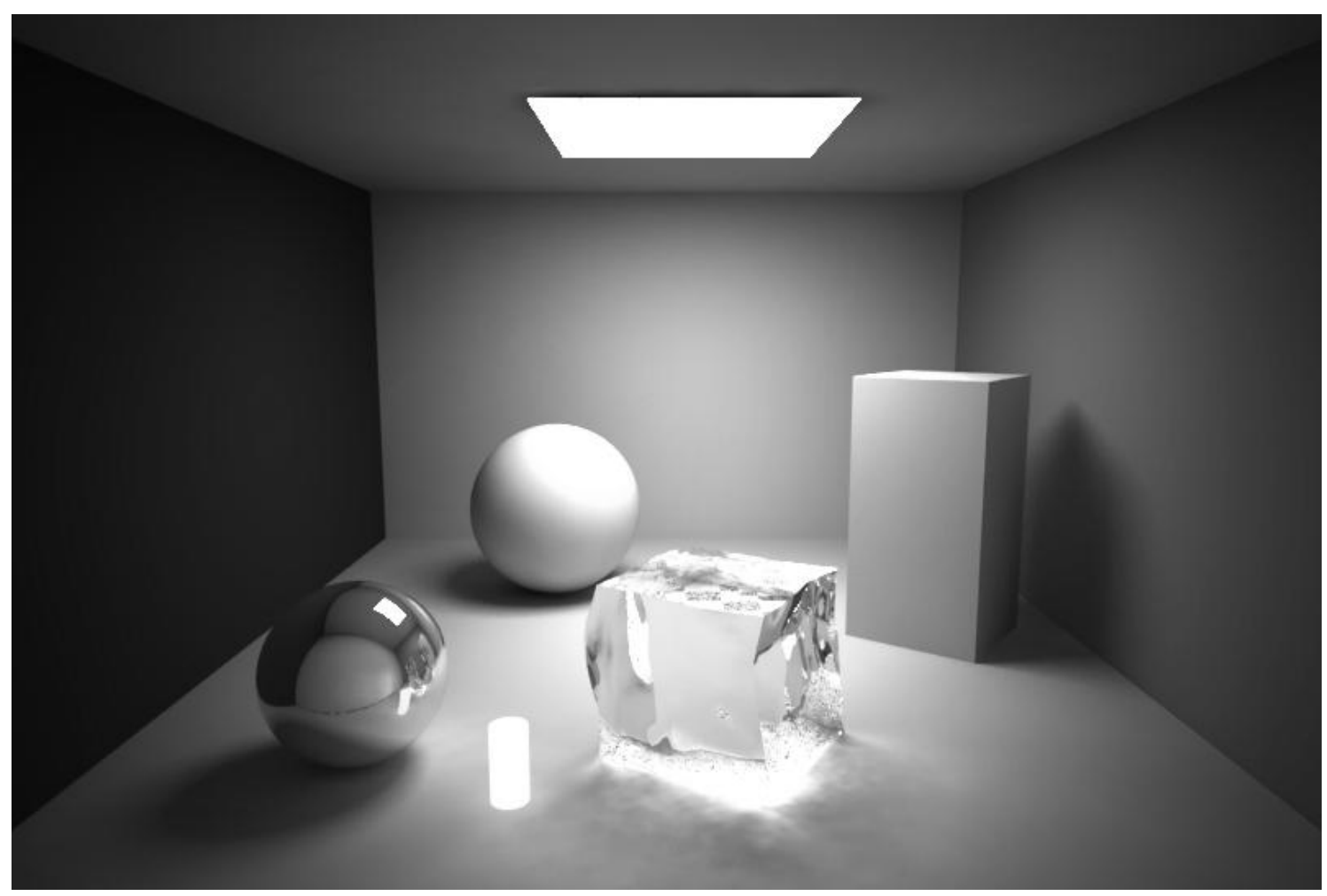

Fig. 1. Virtual image made by computer program for 3D modeling (Skala, 2010) 
and the advanced visualization in science, engineering and art. Each field of application uses a variety of relationships and characteristics of existing techniques and technologies (Boada, 2001).

Using the ray tracing algorithm (Teller \& Hanrahan, 1993) as a technique based on simulating the physical behavior of light using the mathematical tools, in the application Cinema 4D to generate photorealistic images that resemble objects as a result of computer simulations. Using Cinema 4D on the distributed computing environment opens a new field of possibilities in the technology of graphic visualization (Skala et al., 2008). Visualization of virtual objects that do not exist in physical reality, opens a new entries and opportunities in science, engineering and artistic visualization, and is used particularly in the field of engineering and medical sciences, including printing technology. The thesis of this paper is to achieve improvement and optimization of rendering proven performance on new platforms as an extension of the technological foundations of creativity in the field of graphics technology.

One of the problems with which every render system faces is the problem of sampling because each process is trying to show the scene rendering as continuous function in a finite number of pixels (Amburn et al. 1986). If we create a complex scene containing many objects (polygons), and if we modify render parameters (higher or lower values), this rendering process can take a long time. One of the parameters within render settings is a global illumination (GI) and it contains all the relevant algorithms for obtaining the real motion of light rays and lights the scene.

\subsection{Global illumination}

By the definition global illumination is a generic term for a group of algorithms used in 3D computer graphics (Wald, 2004). Some of these algorithms are: radiosity, ray tracing, ambient occlusion, photon mapping. In case of such effects of simulate light the position of the camera, light source, object and their texture and properties are important. For each image pixel, one or more ray is sent from the camera to determine whether the interaction of light rays reflected from some object. Every time when the reflected beam comes to interaction with the object, color of an object in this point is calculated. For this reason, the ray is sent back to each light source to determine the amount of reflected light from the object. These are the so-called "shadow rays" that determines if the point from which came the light lies in the shadow or not. If the surface is reflex or transparent, new rays are sent and tracked to determine the contribution of reflexed or absorbed light of the overall color of the surface. For more complex types of light, such as atmospheric effects or special light is sent to a lot more rays.

The total amount of output light at any point of space is equal to the sum of the emitted light (which may be zero, if there is no source of light) and contributions of all light rays that fall on this point, or may be the result of direct light to other light source or result of (multiple) reflection of light from objects in the scene (Wu et al., 2003). Also, the light is reflected from various objects in a different way. All we can say now formally written as follows: 


$$
L_{o}\left(x, \omega_{o}\right)=L_{e}\left(x, \omega_{o}\right)+\int_{\Omega} \rho_{r}\left(x, \omega_{i}, \omega_{o}\right) L_{i}\left(x, \omega_{i}\right)\left(\omega_{i} \vec{n}\right) d \omega_{i}
$$

Where:

$x$ - point on the scene

$d \omega_{i}$ - differential of the surface at the point $x^{\prime}$

$\omega_{o}$ - outgoing trajectories at the point $\mathrm{x}$

$L_{o}\left(x, \omega_{o}\right)$ - radiance (gloss) in point $\mathrm{x}$ in the direction $\omega_{o}$

$L_{e}\left(x, \omega_{o}\right)$ - transmitting gloss in point $\mathrm{x}$ in the direction $\omega_{o}$

$\rho_{r}\left(x, \omega_{i}, \omega_{o}\right)$ - measures of the light reflected at the surface at the point $\mathrm{x}$, from the output to the incident direction

$\int_{\Omega} \ldots d \omega_{i}$ - integral of the incident beam of light across the hemisphere

$\left(\omega_{i} \vec{n}\right)$ - weakening because of the angle of incident light falls, and $\vec{n}$ is the normal to the surface

Difficulties in obtaining accurate illumination depend on: the degree of dependence between all the surfaces, as the interaction among these surface changes depending on when the system is divided into multiple polygons, and the degree of unpredictability. The first two problems can be considered as complex statistical measurement, which quantify cohesion, structure, or relationship between parts of the system, while the third problem can be considered as entropy, which measures the randomness or unpredictability.

In this work the main point of interest is global illumination and its values that are changed in order to study the length of rendering.

\section{Experimental part}

In the experimental part we take and we render a scene with different settings of global illumination. The scene will be the image instead of the animation for ease of presentation in this paper. Program we used for analyzes the duration is Cinema 4D render. When rendering image, certain parameters were taken into consideration. There are some terms that will be used in the paper, which are part of the simulation of global illumination so before next reading they should be explained.

The irradiance cache (IR) is a collection of points in 3D space (a point cloud) along with the computed indirect illumination at those points. When an object is hit during a GI pass, ray looks into the irradiance cache to see if there are any points similar in position and orientation to the current one. From those already computed points, ray can extract various information (i.e. if there are any objects close by, how fast the indirect illumination is varying etc). Based on that information, ray decides if the indirect illumination for the current point can be adequately interpolated from the points already in the irradiance cache, or not. If not, the indirect illumination for the current point is computed, and that point is stored in the irradiance map. During the 
actual rendering, ray uses a sophisticated interpolation method to derive an approximation of the irradiance for all surfaces in the scene.Quasi Monte Carlo (QMC) is a brute force GI that samples every pixel. It is good for fine detail, but can be slow. It needs high stochastic ray counts to produce grain free results but can produce impressive photorealistic results given enough time. Besides being relatively slow and processor intensive, a principle disadvantage of this method compared to the irradiance cache method is that it writes no cache to disk. Its solution, therefore, unlike with irradiance cache, cannot be saved or reused. IR+QMC creates just a single ray bounce at the collision point of the last ray, so instead of creating a new ray flower for each ray collision, only a single ray is traced through the next pass, so it's much faster with higher pass settings than is IR. Because IR+QMC use more initial rays than IR, it gives a slower but slightly better result than IR when only one ray depth is selected. With higher ray depths, the IR mode uses more stochastic rays at higher depths, so it will be much slower than IR+QMC but give truer results. IR is most suitable for interiors, the extra rays being useful for getting into those nooks and crannies, while IR+QMC is more suited to outdoor renders but can do well indoors too. If speed is a consideration, IR+QMC is your friend with higher diffuse depths.

At the Render Settings of the program, in the tab "General" there is a parameter "GI Mode" and it determines the main settings of the global illumination depending whether there is animation of scenes or images. As we said we take the image settings, especially IR. Another setting that we can change is the "Diffuse Depth" and that instead of the default measure we write , 3 " which means the light will be 3 times reflected from the surface in the scene as opposed to the standard 1. Other parameters in this folder are left unchanged.

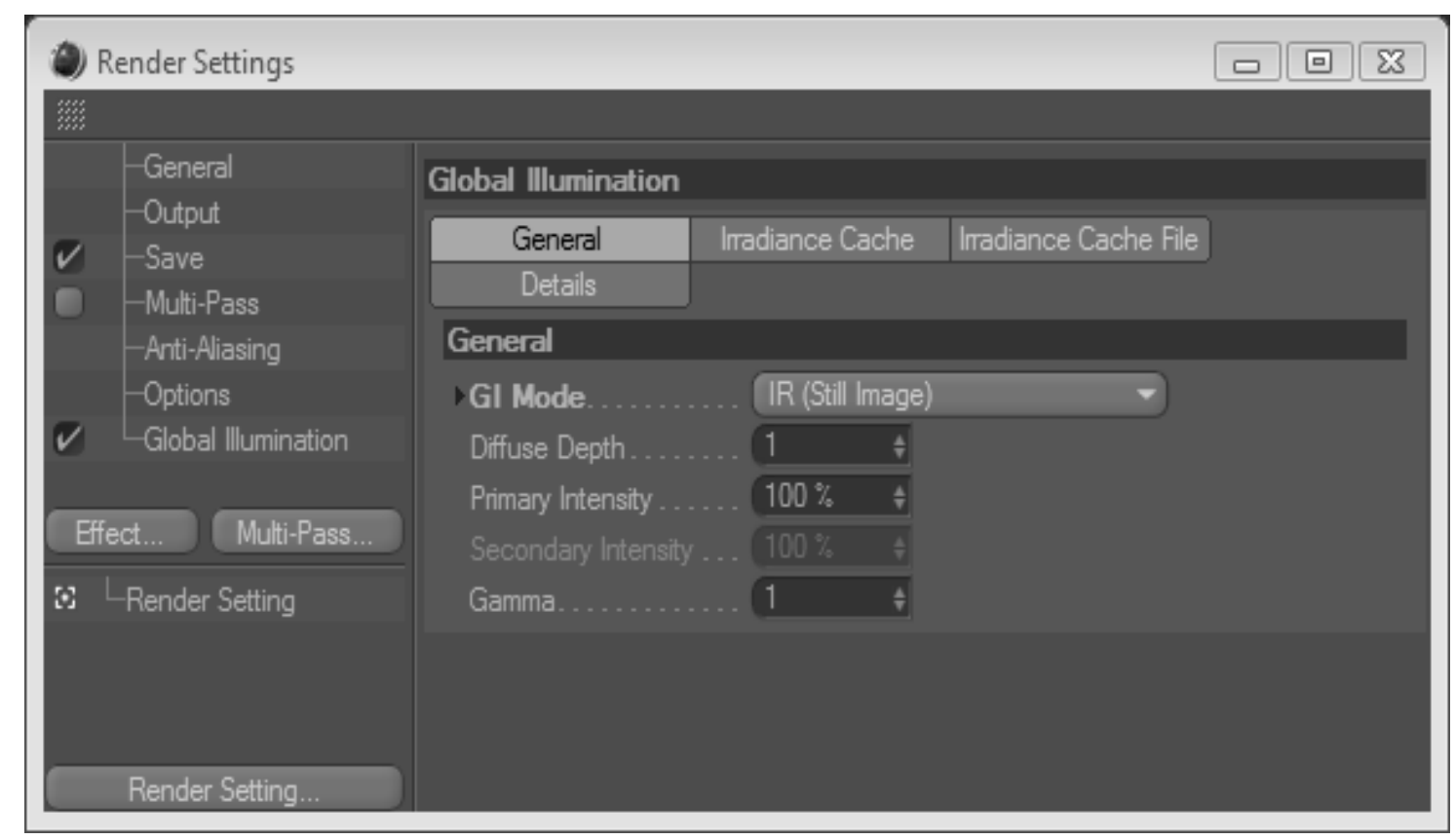

Fig. 2. View of the window Global Illumination at the map General

In another tab called "Irradiance Cache" first parameter is called "Stohastic Samples" in which we set our own preferences to the value of 1500 under "Sample 
Count," which means that it will be 1500 samples per view point at which the light falls. This value during analysis was not changed. „Record Density“ is the next parameter in which we wish to set the resolution at which the light falls. Suppose for the value of 0 each pixel will be analyzed. For the value $-1,4$ pixels will be analyzed, for the value $-2,16$ pixels, etc. Settings were defined as: minimum rate $(-7)$, maximal rate (-2), minimum radius $(10 \%)$ and density control $(10 \%)$. These settings also were not changed. As "Interpolation Method" „Least Squares" was used. For the parameter "Smoothing" initial value "Very Heavy" was set. Same for „Oversempling“. Last setting that was changed was the "Anti-aliasing", which is not part of a Global Illumination.

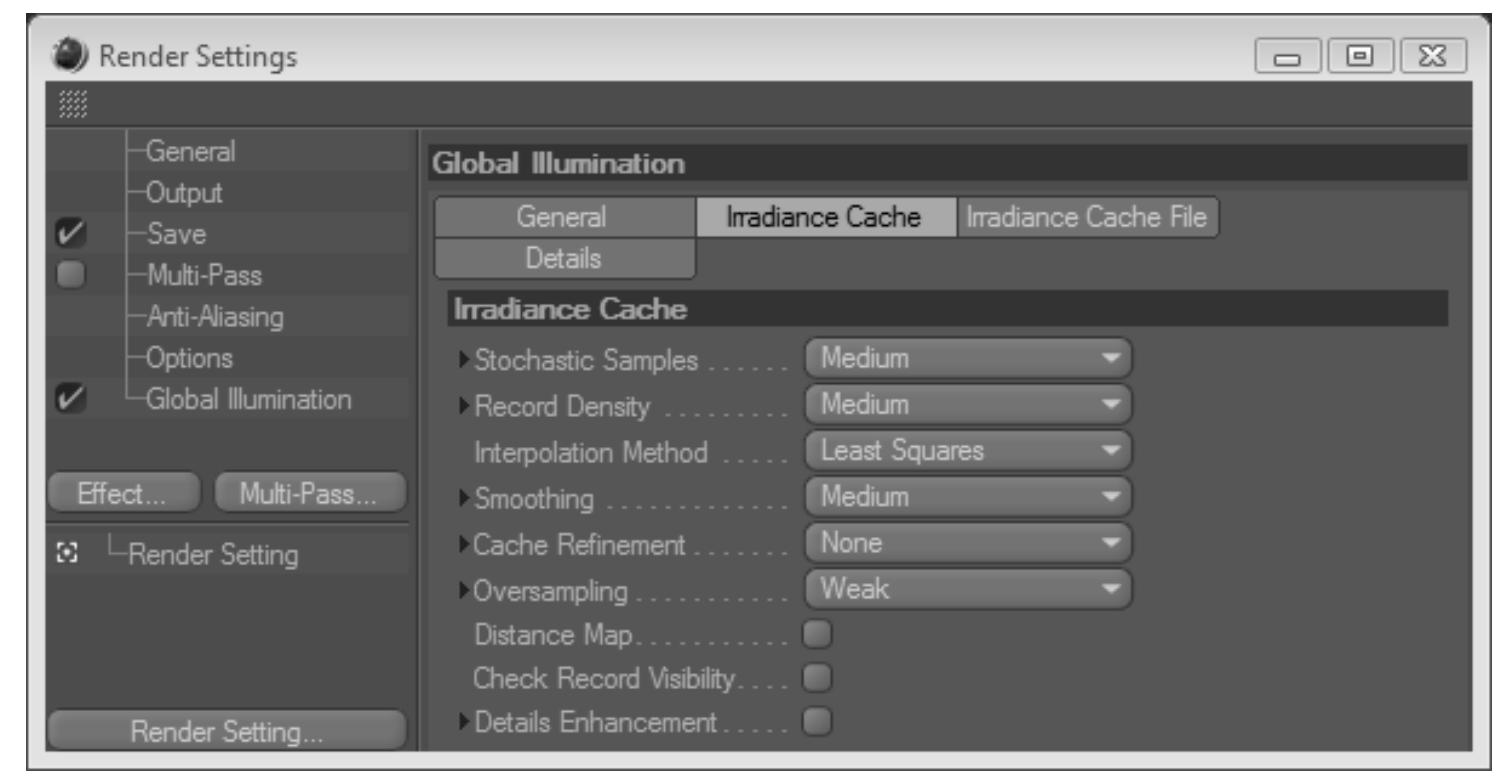

Fig. 3. View of the window Global Illumination at the map Irradiance Cache

\subsection{Analyses of data obtained by measuring}

As it can be seen from the Table 1. time for the rendering of the first measurement with the above settings is 1 hour $52 \mathrm{~min}$ and $50 \mathrm{sec}$. This time represents the highest quality picture we got with our measurements. The next measurement was shorter and lasted 1 hour $37 \mathrm{~min}$ and $50 \mathrm{sec}$ which means that the time has decreased by $13 \%$, but there was no change in picture quality. The reason why render time was

\begin{tabular}{|c|c|c|c|c|c|}
\hline GI mode & IR & QMC +IR & QMC +IR & QMC +IR & QMC +IR \\
\hline Diffuse depth & 3 & 3 & 3 & 3 & 3 \\
\hline $\begin{array}{l}\text { Smoothing } \\
\text { method }\end{array}$ & Very heavy & Very heavy & Very heavy & Medium & Medium \\
\hline Oversempling & Very heavy & Very heavy & Medium & Medium & Minimal \\
\hline Antialiasing & Best & Best & Best & Best & Best \\
\hline TIME & $\begin{array}{c}01 \text { h } 52 \text { min } \\
50 \text { sec }\end{array}$ & $\begin{array}{c}01 \text { h } 37 \text { min } \\
50 \mathrm{sec}\end{array}$ & $\begin{array}{c}01 \mathrm{~h} 23 \mathrm{~min} \\
49 \mathrm{sec}\end{array}$ & $\begin{array}{c}01 \mathrm{~h} 10 \mathrm{~min} \\
45 \mathrm{sec}\end{array}$ & $\begin{array}{c}01 \mathrm{~h} 03 \mathrm{~min} \\
43 \mathrm{sec} \\
\end{array}$ \\
\hline
\end{tabular}

Tab. 1. Data for the first five measurement time rendering 


\begin{tabular}{|c|c|c|c|c|c|}
\hline GI mode & QMC +IR & QMC +IR & QMC +IR & QMC +IR & QMC +IR \\
\hline Diffuse depth & 3 & 3 & 3 & 2 & 1 \\
\hline $\begin{array}{c}\text { Smoothing } \\
\text { method }\end{array}$ & Minimal & Minimal & Minimal & Minimal & Minimal \\
\hline Oversempling & Minimal & Minimal & Minimal & Minimal & Minimal \\
\hline Antialiasing & Best & Geometry & None & None & None \\
\hline TIME & $\begin{array}{c}\mathbf{5 3} \mathbf{~ m i n} \\
\mathbf{5 0} \mathbf{s e c}\end{array}$ & $\begin{array}{c}\mathbf{3 5} \mathbf{~ m i n} \\
\mathbf{5 1} \mathbf{~ s e c}\end{array}$ & $\mathbf{2 5} \mathbf{~ m i n ~ 0 9} \mathbf{~ s e c}$ & $\begin{array}{c}\mathbf{2 1} \mathbf{~} \mathbf{m i n} \mathbf{4 2} \\
\mathbf{s e c}\end{array}$ & $\begin{array}{c}\mathbf{1 8} \mathbf{~ m i n} \\
\mathbf{4 3} \mathbf{~ s e c}\end{array}$ \\
\hline
\end{tabular}

Tab. 2. Data for the five other measurement time rendering

shorter is that IR mode unlike the QMC + IR mode sends a beam of light as many times (on places where light interacted with surface of scene) how much the "Diffuse Depth" is set, in this case 3 times, while the QMC + IR sends just one beam of light in a place where it happened last interaction. The third measure had even shorter render time for an additional $13 \%$, but still there is no change in picture quality. In this measurement we changed "Oversempling" from "Very Heavy" to "Medium", which means that it is used only 16 additional beams on the place where reflection is measured, instead of 256 beams. In the fourth measurement time is shortened by $14 \%$ and the image quality still remained unchanged. "Smoothing" we changed from "Very Heavy" to "Medium" and due to the reduced number of points that serve to

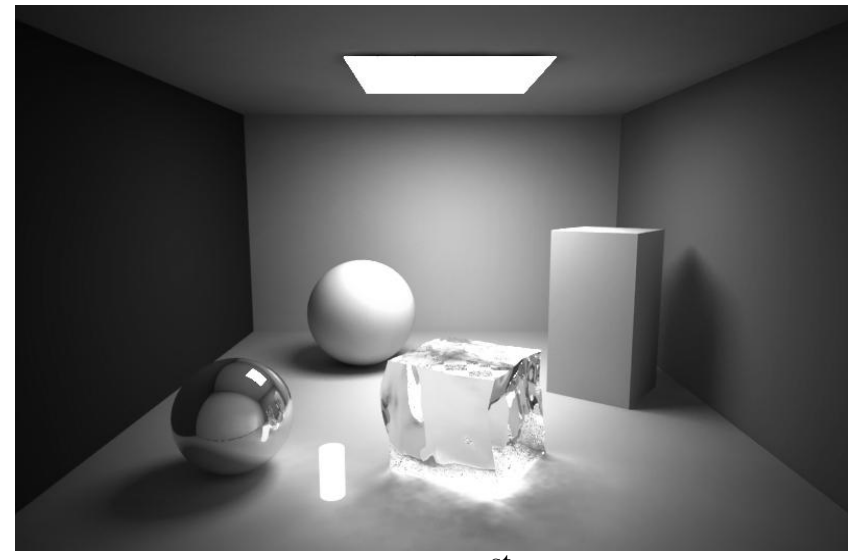

Fig. 4. Image made by $1^{\text {st }}$ measurement

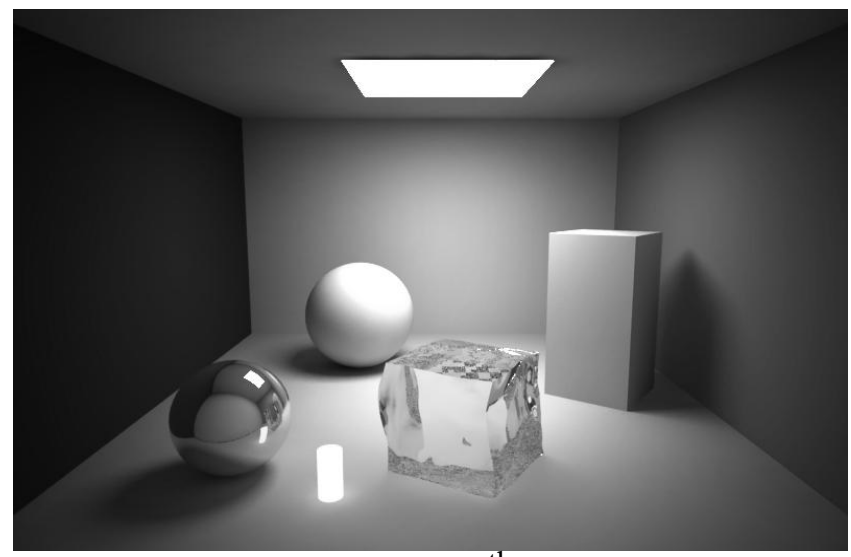

Fig. 6. Image made by $7^{\text {th }}$ measurement

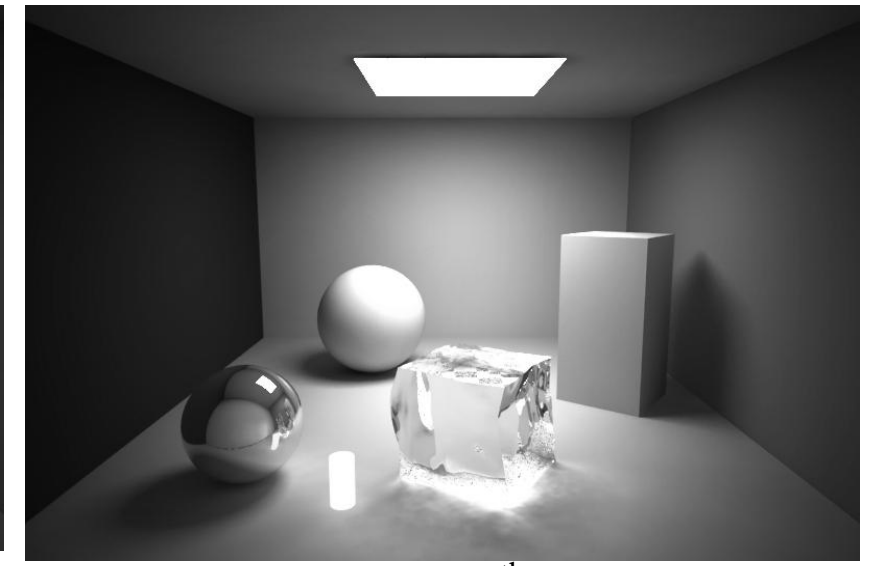

Fig. 5. Image made by $5^{\text {th }}$ measurement

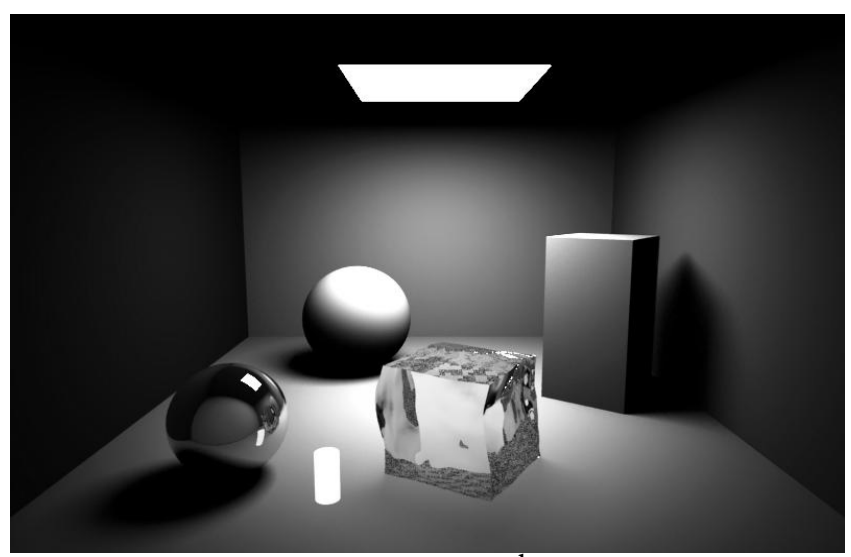

Fig. 7. Image made by $10^{\text {th }}$ measurement 
"smooth" surface of the object the render time is shorter (number of points is reduced from 64 to 32). Fifth measurement recorded less time reduction - 11\%, still without changing the quality. The reason is the same as in the third measure only the number of additional beams decreased from 16 to 4 additional rays. Render time on the next measurement is reduced by $16 \%$, and the reason is the same as in the fourth measurement. In this case, we reduce the number of points from 32 to 16 and the quality remains the same. The seventh measurement was different because we changed the "Anti-aliasing" from the value "Best" to the "Geometry". Rendering time was reduced by as much as $33 \%$. But there can be discerned in the first signs of lower quality. In certain parts of the image appears a sense of "moire" and at zoomed parts of the figure can jaggy easily be noticed. Here we can see that these setting affected very little on image quality. It tries to show the object with as smoother edges as possible. The difference between "Best" and "None" is similar to the vector line in Adobe Illustrator and raster line in Adobe Photoshop. Modifying the "Diffuse Depth", from 3 to 2 , in the ninth measurement we got a reduction in time by $12 \%$ and from 2 to 1 , in the tenth measurement, it was reduced by $13 \%$.

\section{Conclusion}

Through this work research in the field of multimedia communications with thematic focus on 3D complex graphical content rendering was carried out. In this area is a distinct expansion of the speed of transmission and processing of image elements with the help of using modern and advanced parallel computing software. The work was done to optimize and find the best ratio of quality and rendering time of complex graphic structure. If the complexity of the image (defined by the number of polygons in the scene) is calculating, it is possible to set the appropriate settings so that the time required for rendering could get shortened. This work was desired to find a solution and provide guidance and instructions for scientists who uses 3D graphics without losing time on the settings that are not important and does not affect too much on image quality alone adjusted parameters of your applications.

The experimental part was performed on the 3D modeled graphics structure created for testing and described in this paper that the scene contained a number of different models and thus constitute a complex graphics scene. Reducing and changing the parameters and render settings after a large number of measurements on the sample we have found the optimum ratio to reduce processing time. The limitations of our work are quite complex graphic structure that needs lot of time to render. Also one node is not enough to make render procedure in a time, so basically distributed system would be better solution. Our next research will be on the way of how to make complex computer animations look photorealistic and render them in as less time as possible. Computer graphics and 3D modeling are extremely complex topic areas still unexplored, and this work is at least partly enabled better insight into the question of reducing the timing of rendering.From this it is clear that this work includes the modern technological area in which this is a new technological chain and the pragmatic new field in graphic technology. The focus of research activities related 
Skala, T.; Muza, R. \& Mrvac, N.: Render Settings Impact Analyses on Quality of ...

to an optimum method of acceleration process of generating image content using render settings on 3D modeling application.

\section{References}

Amburn, P. ; Grant, E. \&Whitted, T. (1986). Managing geometric complexity with enhanced procedural models. Conference Proceedings Computer Graphics, 20(4):189-195, August 1986. ACM Siggraph '86

Arvo, J. R.; Analytic (1995).Methods For Simulated Light Transport; Ph.D. dissertation, Yale University

Boada, I.; Navazo, I. \& Scopigno, R. (2001). Multiresolution volume visualization with a texture-based octree. The Visualizated Computer

Gortler, S. J.; Schroeder, P.; Cohen, M. F. \& Hanrahan, P.(1993). Wavelet radiosity. Computer Graphics (Proceedings of SIGGRAPH'93), 27:221-230, August 1993. Held in Anaheim (CA), USA.

Rigau, J.; Feixas, M. \& Sbert, M; (2000). Visibility complexity of animation in flatland. Research Report IIiA-00-05-RR, Institut d'Informatica i Aplicacions, Universitat de Girona, Girona, Spain

Rigau, J.; Feixas, M..; Bekaert, P. \& Sbert, M.; (2001). View-dependent information theory measures for pixel sampling and scene discretization in atland. In Proceedings of Spring Conference on Computer Graphics'01, 173-180, Los Alamitos (CA), USA, April 2001. IEEE Computer Society. Held in Budmerice, Slovak Republic

Rigau, J.; Feixas, M.. \& Sbert, M.; (2002). New Contrast Measures for Pixel Supersampling, Proceedings of CGI'02, Bradford, UK, 439-451. SpringerVerlag London Limited, London, UK, July 2002.

Skala, T.; Mrvac, N.; Mikota, M. \& Pavlović, I..(2008). Multimedia Image Rendering on a Distribued Computer System. DAAAM International Scientific Book 2008. Katalinic, Branko. Vienna: DAAAM International, 2008: 781-788

Skala, T. (2010). Effectiveness of the procedure of rendering graphic content on a distributed computing system; Ph.D. dissertation, University of Zagreb

Teller, S. J. \& Hanrahan, P. (1993) Global visibility algorithms for illumination computation. Computer Graphics.Proceedings of SIGGRAPH'93, 27:239-246, August 1993. Held in Anaheim (CA), USA

Wald, I. \& Slusallek, P. (2001). State of the Art in Interactive Ray Tracing; Computer Graphics Group, Saarland University

Wald, I. (2004). Realtime Ray Tracing and Interactive Global Illumination; Dissertation zur Erlangung des Grades Doktor der Ingenieurwissenschaften, Naturwissenschaftlich Technischen Fakultät I der Universität des Saarlandes

Wu, Y.; Bhatia, V.; Lauer, H. \& Seiler, L. (2003). Shear-image ray-casting volume rendering. ACM SIGGRAPH Symposium on Interactive 3D Graphics 